\title{
Der erste Schritt ist der schwerste
}

Angehörige psychisch Kranker bleiben bei der Pflege oft selbst auf der Strecke, sind verzweifelt, wütend und traurig. Sich einzugestehen, dass man selbst Hilfe braucht, kostet Überwindung. Lesen Sie in unserem Fallbeispiel, wie die Mutter einer psychisch Erkrankten durch eine Selbsthilfegruppe neue Kraft fand. Um den entscheidenden Schritt aus der Anonymität zu wagen, half ihr eine Wanderung.

Ingrid Langschwert

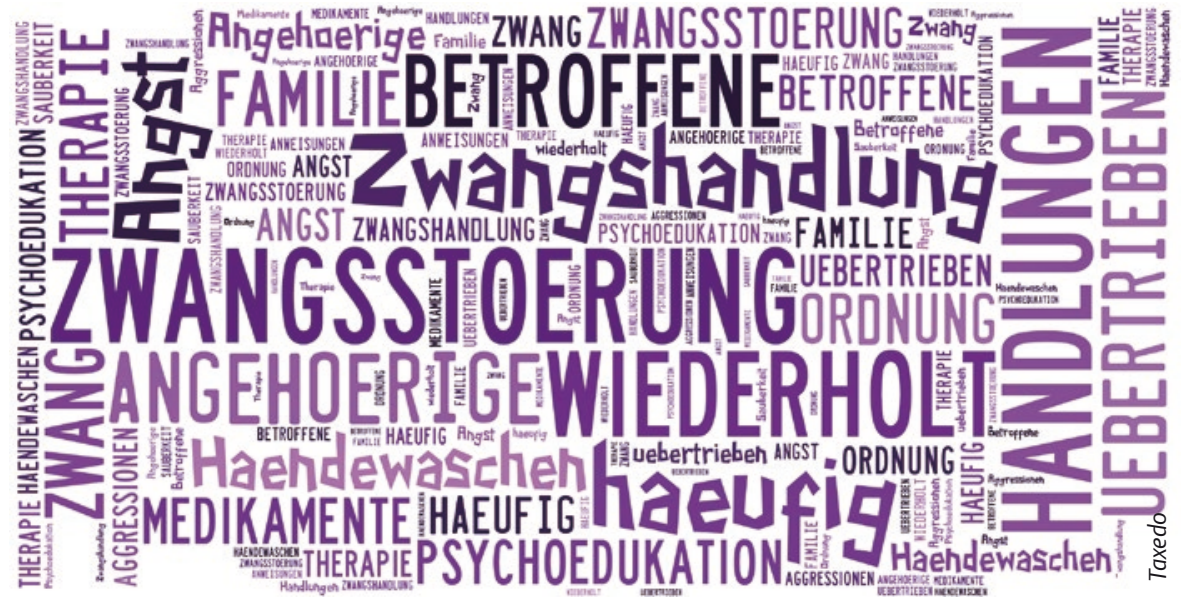

Wäre alles anders gekommen, hätte sie die ersten Zeichen richtig gedeutet? Die Dame mir gegenüber in der Geschäftsstelle des Landesverbands Bayern der Angehörigen psychisch Kranker $(\rightarrow$ Infokasten) zuckt die Schultern. Diese Frage hat sie sich schon oft gestellt. Eine Antwort hat sie bislang nicht gefunden.

Heute ist ihre einst so vielversprechende Tochter 28 Jahre alt. Sie hat die Schule abgebrochen und arbeitet nicht, obwohl ihr äußerlich nichts zu fehlen scheint. Eileen hat eine eigene Wohnung. Dennoch lebt sie häufig bei ihren Eltern.

Eileen leidet unter einer Zwangsstörung. Sie braucht jeden Tag um dieselbe Zeit eine bestimmte Pizza aus einem Geschäft, das eineinhalb Stunden vom Wohnort entfernt ist - etwas anderes kann sie abends nicht zu sich nehmen. Was wie Extravaganz aussieht, ist eine Zwangsstörung, die sich bei Betroffenen und Angehörigen zur Tragödie auswachsen kann. Eileens Eltern befürchten, dass ihre Tochter verhungern könnte.
Zwischen drei und vier Prozent der deutschen Bevölkerung erkranken einmal im Leben an einer Zwangsstörung. Diese Menschen wissen, dass ihre Handlungen sinnlos, oft sogar schädlich sind, können aber ihren Impuls, die Handlungen dennoch auszuüben, nicht unterdrücken. Zwangserkrankte Menschen fallen selten auf, weil sie ihre Wohnung kaum verlassen (können), oder weil sich das zwanghafte Verhalten nur auf die eigenen vier Wände bezieht.

Kein Wunder also, dass Zwangserkrankungen bis Mitte der 1990er-Jahre so gut wie unbekannt waren. Noch heute kann es bis zu zehn Jahre dauern, bis diese Krankheit diagnostiziert wird. Auch Eileen konnte bislang nicht geheilt werden, obwohl ihre Eltern früh erkannten, dass sie ein besonderer Mensch ist.

\section{„Jetzt weiß ich es besser.“}

Das Gesicht der Frau mir gegenüber erhellt sich, als sie sich erinnert, wie sehr sich ihre Tochter im Kindergartenalter über neue Kleidung freute und wie ordentlich sie alles behandelte. „Jetzt weiß ich es besser“, fährt die Dame traurig fort. Damals hätte sie sich gefreut, als ihre Tochter den Sitzplatz der Oma gegen einen unachtsamen Besucher verteidigte: „Das ist Omas Sessel!“.

Als Eileen allerdings mit neun Jahren beschloss, sich im Gang umzuziehen, damit die Straßenkleider nicht die Wohnung beschmutzten, suchen die Eltern Hilfe. „Ein Tic, das legt sich von selbst“, versicherten ihr Freunde und Nachbarn. Auch die zurate gezogenen Fachleute beruhigten: „Kinder sind sich ihrer Selbstwirksamkeit noch nicht bewusst“. Eileens befremdliches Verhalten sei eine „völlig normale Stressreaktion“.

Noch heute gelten Zwangshandlungen von Kindern unter elf Jahren als harmloses Symptom der Angst- oder Stressbewältigung. „Bedenklich“ sei nur, so die Schweizerische Gesellschaft für Zwangsstörungen, „wenn das Kind einen übermäßig bedrückten Eindruck macht, sich von seinen Freunden und der Familie zurückzieht, stundenlang mit scheinbar unsinnigen Handlungen beschäftigt und insgesamt nicht mehr zugänglich“ sei.

Eileen ging gerne zur Schule, schrieb gute Noten und pflegte intensive Freundschaften. Als sie zwölf Jahre alt wurde, legte sich der Umkleidewahn tatsächlich. Doch Eileen benahm sich deshalb nicht weniger seltsam: Nun schien Nahrung gefährlich zu sein, Verpackungen kontaminiert, das sich darin befindliche Essen vergiftet.

Eileen bekam eine Spieltherapie verschrieben. Trotzdem blieb sie morgens immer häufiger zuhause, denn wenn sie nach der Schule nach Hause kam, war sie sich nie sicher, ob sich ihre Familie an die Sicherheitsvorschriften gehalten hat: Zwei 
Mal hatte sie bereits die Verpackung eines Schokoriegels im Müll gefunden. Tagelang konnte sie keinen Bissen herunterbringen.

\section{Das Zusammenleben wurde unerträglich}

Als Eileen 16 Jahre alt war, beschloss sie, das Haus gar nicht mehr zu verlassen und nicht mehr zur Schule zu gehen. Der Therapeut, zu dem Eileen nun gebracht wurde, beendete die Behandlung nach 20 Stunden. In seinen Augen war Eileen spätpubertär und testete nur die Grenzen der Eltern.

Die 17-jährige Eileen litt darunter, dass ihr Verhalten der Familie schadete. Ihr jüngerer Bruder kam nur noch zum Schlafen nach Hause und ihre Eltern schanzten sich gegenseitig die Schuld zu. Eileen hoffte nun auf die Hilfe einer Klinik. Dort erhielt sie Medikamente, Therapie und Psychoedukation. Die Medikamente und die Therapie brachten wenig, wohl aber die Psychoedukation - und die Liebe.

Franz, den sie in der Klinik kennenlernte, litt ebenfalls unter Zwängen. Mit der Unterstützung der Eltern fanden sie eine gemeinsame Wohnung. Er kaufte nach Anweisung ein, sie ertrug seine Gebete und seine Einteilung der Menschen in Dämone und Heilige. Sie sind glücklich - bis Eileen in die Kategorie „Dämon“ rutscht.

\section{$\rightarrow$ LANDESVERBAND BAYERN DER ANGEHÖRIGEN PSYCHISCH KRANKER E. V.}

Der Landesverband Bayern der Angehörigen psychisch Kranker e. V. ist die gemeinnützige Dachorganisation der bayerischen Angehörigen-Selbsthilfe. Der Landesverband besteht seit 1990 und hat derzeit 2350 Mitglieder.

Er ist parteipolitisch und konfessionell neutral und wird von den bayerischen Bezirken und von der Selbsthilfeförderung der Krankenkassen finanziell unterstützt. Er ist Mitglied im Bundesverband der Angehörigen psychisch Kranker e. V. mit Sitz in Bonn.

All seine Anstrengungen haben zum Ziel, die Lebensqualität der Angehörigen psychisch kranker Menschen zu verbessern, ihnen Benachteiligungen zu ersparen und die Familien im selbstbewussten Umgang mit dem Schicksal einer psychischen Erkrankung zu stärken.
Sie zog wieder zu Hause ein. Ihre Zwänge nahmen zu. Ihre Aggressivität ebenfalls. Wurden ihre Anweisungen nicht buchstabengetreu ausgeführt, drehte sie durch. Täglich gab es Streit. Manchmal eskalierte er. Eines Tages riefen die Eltern die Polizei. Die führte ihr Kind ab. Der Blick, den Eileen ihnen da zuwarf, werden sie nie vergessen. In der Klinik wurde Eileen fixiert. Als ihr Widerstand gebrochen war, wird sie entlassen, denn die Zwänge traten im Klinikalltag nicht auf.

Eileen wollte nicht zurück in die Wohnung, die sie zusammen mit Franz bewohnt hatte. Sie zog wieder zu ihren Eltern. Alle nahmen sich zusammen, doch die nächste Eskalation war vorprogrammiert. Eileen ging freiwillig in eine Klinik. Die Therapien schienen anzuschlagen. Eileen konnte sich öffnen und machte Fortschritte. Nach drei Monaten Klink war Schluss. Die Krankenkasse hätte weiter gezahlt. Doch der Arztbrief mit der Begründung für eine Verlängerung kam zu spät.

Eileen stand wieder vor der elterlichen Tür - nur dass die Wohnung inzwischen kleiner geworden war, denn die Zuzahlungen für die Therapien und Ärzte hatten Spuren beim Ersparten der Eltern hinterlassen.

In der neuen Wohnung wurde das Zusammenleben mit dem erkrankten Kind unerträglich. Die Frau mir gegenüber kommt an ihren Tiefpunkt: Sie hatte alles in ihrer Macht stehende versucht, um ihr Kind vom Zwang zu befreien. Jetzt brauchte sie selber Hilfe, um weiterhin für den Erhalt der Restfamilie sorgen zu können.

\section{Verständnis und Austausch}

Erstmals suchte die Mutter Hilfe für sich und fand sie in Form einer Informationsveranstaltung des Landesverbands Bayern der Angehörigen psychisch Kranker e.V. Dort traf sie auf Menschen, die ähnliches Leid erfahren haben und die nicht daran zerbrochen sind. Das Angebot einer kostenlosen Einzelberatung nahm die Mutter deshalb gerne an. Sie bekam die Empfehlung, sich einer örtlichen Selbsthilfegruppe anzuschließen - und die Einladung zu einer gemeinsamen Wanderung.

Den Besuch bei der Selbsthilfegruppe schlug die Dame zunächst aus. An der Wanderung nahm sie aber teil. Da gab es Menschen, wie sie, Menschen, die eine lan- ge Leidensgeschichte hinter sich hatten, aber trotzdem herzhaft lachen konnten, und Menschen, die sie nicht für die Krankheit ihrer Tochter verantwortlich machten.

„Deshalb ging ich eines Tages doch zu so einem Gruppentreffen“, setzt die Dame ihre Geschichte fort, obwohl sie Angst hatte, von Fremden kritisiert zu werden. Doch die acht Männer und Frauen, die sich an dem Abend versammelt hatten, zeigten nur Verständnis. Sie kannten ähnliche Situationen, ähnliche Gefühle.

Egal, wie das Krankheitsbild des Betroffenen genannt wird, die Gefühle und Probleme, vor die dessen Familie gestellt wird, sind dieselben: Angst, Verzweiflung, Wut, Trauer. Und Fragen, wie: Wie viel Verantwortung kann und sollte der Betroffene selbst übernehmen? Welche Hilfen und welche Unterstützungsangebote gibt es? Wie lassen sich die Hilfen finanzieren?

Nach ihrem ersten Besuch in der Gruppe war der Dame klar: Sie wird wiederkommen. Sich eingestehen, dass man selbst Hilfe braucht, ist der entscheidende erste Schritt. Erst später wird die Dame erkennen, dass sie an der Krankheit ihrer Tochter gewachsen ist und neue Fähigkeiten entwickelt hat, die sie eines Tages positiv einsetzen kann. Doch das ist - noch Zukunftsmusik.

PS: Eileen gibt es nicht. Ihre Geschichte und die ihrer Familie basiert aber auf realen Beratungsfällen des Landesverbands Bayern der Angehörigen psychisch Kranker e.V., München. Zum Schutz der betroffenen Personen sind die Fälle zur Unkenntlichkeit verfremdet.

\begin{tabular}{l}
\hline AUTORIN \\
Ingrid Langschwert \\
Referentin für Öffentlichkeits- \\
arbeit im Landesverband Bayern \\
der Angehörigen psychisch \\
Kranker(LApK) e.V. \\
E-Mail: LApK@mpm- \\
langschwert.de
\end{tabular}

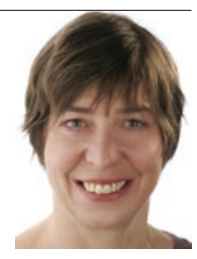

langschwert.de

\section{BIBLIOGRAFIE}

DOI 10.1055/s-0041-106268

Psych Pflege 2015; 21: 288-289

(c) Georg Thieme Verlag KG

Stuttgart · New York · ISSN 0949-1619 